\title{
Edge Elimination in TSP Instances
}

\author{
Stefan Hougardy and Rasmus T. Schroeder \\ Research Institute for Discrete Mathematics, University of Bonn
}

\begin{abstract}
The Traveling Salesman Problem is one of the best studied NP-hard problems in combinatorial optimization. Powerful methods have been developed over the last 60 years to find optimum solutions to large TSP instances. The largest TSP instance so far that has been solved optimally has 85,900 vertices. Its solution required more than 136 years of total CPU time using the branch-and-cut based Concorde TSP code [1. In this paper we present graph theoretic results that allow to prove that some edges of a TSP instance cannot occur in any optimum TSP tour. Based on these results we propose a combinatorial algorithm to identify such edges. The runtime of the main part of our algorithm is $O\left(n^{2} \log n\right)$ for an $n$-vertex TSP instance. By combining our approach with the Concorde TSP solver we are able to solve a large TSPLIB instance more than 11 times faster than Concorde alone.
\end{abstract}

Keywords: traveling salesman problem, exact algorithm

\section{Introduction}

An instance of the Traveling Salesman Problem (TSP for short) consists of a complete graph on a vertex set $V$ together with a symmetric length function $l: V \times V \rightarrow \mathbb{R}_{+}$. A tour $T$ is a cycle that contains each vertex of the graph exactly once. The length of a tour $T$ with edge set $E(T)$ is defined as $\sum_{e \in E(T)} l(e)$. A tour $T$ for a TSP instance is called optimum if no other tour for this instance has smaller length. Finding such an optimum TSP tour is a well known NP-hard problem [5.

The Traveling Salesman Problem is one of the best studied problems in combinatorial optimization. Many exact and approximate algorithms have been developed over the last 60 years. In this paper we present several theoretical results that allow us to eliminate edges from a TSP instance that provably cannot be contained in any optimum TSP tour. Based on these results we present a combinatorial algorithm that identifies such edges. As the runtime of our main algorithm is only $O\left(n^{2} \log n\right)$ for an $n$-vertex instance, it can be used as a preprocessing step to other TSP algorithms. On large instances our algorithm can speed up the runtime of existing exact TSP algorithms significantly. It also can improve the performance of heuristic algorithms for the TSP. We present examples for both applications in Section 7 For a good description of the state of the art in algorithms for the Traveling Salesman Problem see [1].

Our results are motivated by studying 2-dimensional Euclidean TSP instances, i.e., instances where the vertices are points in the Euclidean plane and 
the length of an edge is the Euclidean distance between the two corresponding points. However, most of our results hold for arbitrary symmetric TSP instances that even do not need to be metric.

Our Contribution. We present several results that allow to prove that certain edges in a TSP instance cannot belong to any optimum TSP tour. Our Main Edge Elimination Theorem that we prove in Section 3 turns out to be quite powerful. It allows to reduce the $n(n-1) / 2$ edges of an $n$-vertex TSP instance to about $30 n$ edges or less for the TSPLIB [9] instances. Some additional methods for eliminating edges are presented in Section 4. We combine these with a backtrack search which we present in Section 6. This will allow us to reduce the number of edges in the TSPLIB instances to about $5 n$ edges.

Unfortunately, our Main Edge Elimination Theorem does not directly lead to an efficient algorithm for eliminating edges in a TSP instance. Thus, a second major contribution of this paper is contained in Section 5 where we prove that a weaker form of our Main Edge Elimination Theorem can be checked in constant time per edge. This allows to apply our methods to very large TSP instances containing 100,000 or more vertices. The total runtime on such a large instance is less than three days on a single processor. Our algorithm can be run in parallel on all edges resulting in a runtime of less than a minute if sufficiently many processors are available.

Section 7 contains the results of our algorithm on TSPLIB [9] instances as well as on a 100,000 vertex instance. Here we also show how our approach can speed up finding optimum solutions to large TSP instances significantly. The TSP solver Concorde [1] is the fastest available algorithm to solve large TSP instances optimally. Concorde needs more than 199 CPU days for the TSPLIB instance d2103. After running our edge elimination algorithm for 2 CPU days the runtime of Concorde decreases to slightly more than 16 CPU days. The total speed up we obtain is more than a factor of 11 .

We also report two other successful applications of the edge elimination approach in Section 7.

\section{Notation and Preliminaries}

To avoid some degenerate cases we assume in this paper that a TSP instance contains at least four vertices. Edges that do not belong to any optimum TSP tour will be called useless. We extend the definition of a TSP instance to instances $(V, E)$ together with a length function $l: E \rightarrow \mathbb{R}_{+}$where $E$ contains all edges of the complete graph on $V$ except some useless edges. This implies that all optimum TSP tours on the complete graph on $V$ are contained in the graph $(V, E)$. Therefore, to find an optimum TSP tour it suffices to consider edges from $E$. However, to prove that some edge in $E$ is useless it might be useful to also look at edges that are not in $E$. Our edge elimination algorithm will start with some TSP instance $(V, E)$ and return an instance $\left(V, E^{\prime}\right)$ such that $E^{\prime}$ is a subset of $E$ and contains all optimum TSP tours. 
As most of our results are inspired by studying Euclidean instances we will often call a vertex in a TSP instance a point. If $x$ and $y$ are two vertices in a TSP instance we will denote the edge $\{x, y\}$ by $x y$ simply.

A TSP instance is called metric if $l(x y) \leq l(x z)+l(z y)$ for all vertices $x, y, z \in V$. Our results do not require that the TSP instance is metric. However, we can improve some of our results if we assume some metric properties of the instance. Euclidean TSP instances are of course metric, but because of problems with floating point accuracy such instances are usually not considered in practice. The well known TSPLIB 9] instances for example use the discretized Euclidean distances EUC_2D and CEIL_2D. In the first, the Euclidean distance is rounded to the nearest integer while in the second it is rounded up to the next integer. Note that the EUC_2D distance function is not metric and that an optimum TSP tour for such an instance may contain two crossing edges.

Currently, the most successful heuristic TSP algorithms [7] are based on the concept of $k$-opt moves. Given a TSP tour a $k$-opt move makes local changes to the tour by replacing $k$ edges of the tour by $k$ other edges. For a $k$-opt move we require, that after the replacement of the $k$ edges the new subgraph is 2regular. If the new subgraph is again a tour we call the $k$-opt move valid. If a tour $T$ allows a valid $k$-opt move resulting in a shorter tour, then $T$ cannot be an optimum tour. This simple observation is the core of our algorithm for proving the existence of useless edges.

Let $p q$ and $x y$ be two edges in a TSP instance. We call $p q$ and $x y$ compatible, denoted by $p q \sim x y$, if

$$
\max (l(p x)+l(q y), l(p y)+l(q x)) \geq l(p q)+l(x y) .
$$

Otherwise $p q$ and $x y$ are called incompatible. Note that two edges that have at least one vertex in common are always compatible.

Lemma 1. Any two edges in an optimum TSP tour are compatible.

Proof. Assume $p q$ and $x y$ are two incompatible edges in an optimum TSP tour $T$. By (11) we have $l(p x)+l(q y)<l(p q)+l(x y)$ and $l(p y)+l(q x)<l(p q)+l(x y)$. Thus $T$ can be improved by a 2-opt move, that replaces edges $p q$ and $x y$ by either $p x$ and $q y$ or by $p y$ and $q x$. One of these two 2-opt moves must be valid. This contradicts the assumption that $T$ is an optimum TSP tour.

For $k>2$ we call a set of $k$ edges $k$-incompatible, if they cannot belong to the same optimum TSP tour.

\section{The Main Edge Elimination Theorem}

To be able to formulate our Main Edge Elimination Theorem, we need to introduce the concept of potential points first. Let $(V, E)$ be a TSP instance and $p q \in E$. For $r \in V \backslash\{p, q\}$ define

$$
R:=\{x \in V \mid r x \in E \wedge p q \sim r x\} .
$$


Let $R_{1}, R_{2} \subset V$ and $R \subset R_{1} \cup R_{2}$. We call $r$ potential with respect to $p q$ and $R_{1}$ and $R_{2}$, if for every optimum tour containing $p q$, the two neighbors of $r$ cannot both lie in $R_{1}$ respectively $R_{2}$. We say that such a covering certifies the potentiality of $r$. Note that $R_{1} \cap R_{2}$ needs not to be empty. However if $r$ is potential, in any optimum tour containing $p q, r$ cannot be connected with $R_{1} \cap R_{2}$. In Section 5 we will develop efficient methods for certifying potential points.

Theorem 1 (Main Edge Elimination Theorem). Let $(V, E)$ be a TSP instance and $p q \in E$. Let $r$ and $s$ be two different potential points with respect to $p q$ with covering $R_{1}$ and $R_{2}$ respectively $S_{1}$ and $S_{2}$. Let $r \notin S_{1} \cup S_{2}$ and $s \notin R_{1} \cup R_{2}$. If

$$
l(p q)-l(r s)+\min _{z \in S_{1}}\{l(s z)-l(p z)\}+\min _{y \in R_{2}}\{l(r y)-l(q y)\}>0
$$

and

$$
l(p q)-l(r s)+\min _{x \in R_{1}}\{l(r x)-l(p x)\}+\min _{w \in S_{2}}\{l(s w)-l(q w)\}>0,
$$

then the edge $p q$ is useless.

Proof. Assume that the edge $p q$ is contained in an optimum TSP tour $T$. Let $r x, r y, s z, s w \in T$ be the incident edges of $r$ and $s$. We may assume that the vertices $x, y, z$, and $w$ are labeled in such a way that $x \in R_{1}, y \in R_{2}, z \in S_{1}$, and $w \in S_{2}$. As $r$ and $s$ are potential, we have $r, s \notin\{p, q\}$. By assumption we have $r s \notin T$, making the four edges $r x, r y, s z$ and $s w$ distinct.

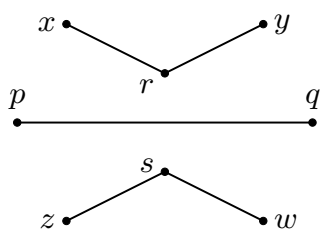

The situation of Theorem 1

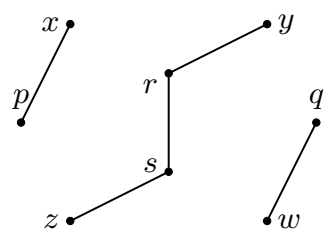

One possible 3-opt.

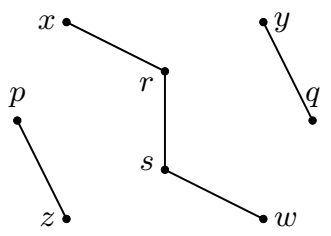

Another possible 3-opt.

Fig. 1. Two possible 3-opt moves that imply that the edge $p q$ is useless.

Now there exist two possible 3-opt moves as shown in Figure 1. The first is to replace $p q, r x$, and $s w$ with $p x, r s$, and $q w$. The second is to replace $p q, r y$, and $s z$ with $p z$, rs, and $q y$. It is easy to verify that for every tour containing the egdes $p q, r x, r y, s z$ and $s w$, one of these two 3-opt moves must be valid.

The two 3-opt moves are decreasing the length of the tour $T$ by

$$
\begin{aligned}
& l(p q)-l(r s)+l(r x)-l(p x)+l(s w)-l(q w) \text { respectively } \\
& l(p q)-l(r s)+l(r y)-l(p y)+l(s z)-l(q z) .
\end{aligned}
$$

By inequalities (3) and (4), both terms are strictly positive. Since one of these 3opt moves is valid, this yields a tour shorter than $T$, contradicting the optimality of $T$. 


\section{The Close Point Elimination Theorems}

The Main Edge Elimination Theorem will be our primary tool to prove that an edge in a TSP instance is useless. As soon as many edges of a TSP instance are known to be useless other methods can be applied. In this section we present our so called Close Point Elimination. When applied to the complete graph of a TSP instance it will eliminate almost no edge. However, in combination with the Main Edge Elimination Theorem it will allow to identify additional useless edges.

Theorem 2 (Close Point Elimination Theorem). Let (V,E) be a TSP instance and $p q \in E$. Let $r \in V \backslash\{p, q\}$ and define $R:=\{x \in V \mid r x \in E \wedge p q \sim r x\}$. If for all $x, y \in R$ with $\{x, y\} \neq\{p, q\}$ we have

$$
l(x y)+l(p r)+l(q r)<l(p q)+l(x r)+l(y r)
$$

then the edge $p q$ is useless.

Proof. Assume that an optimum tour $T$ contains the edge $p q$. Let $x r$ and $r y$ be the two edges in $T$ that are incident with $r$. Then $\{x, y\} \neq\{p, q\}$ and $x$ and $y$ must be in $R$. By assumption inequality (7) holds. Then we can replace the edges $p q, x r$, and $y r$ with $x y, p r$, and $q r$ and obtain a tour that is shorter than $T$. Note that this also holds if one of $x$ and $y$ equals one of $p$ and $q$. This contradicts the optimality of the tour $T$.

For the degenerate case with $x=p$ we show a stronger result by making use of the notion of metric excess. It will allow us to short cut a eulerian subgraph in an instance that needs not to be metric. The metric excess $m_{p q}(z)$ of a vertex $z$ with respect to an edge $p q$ is defined as

$$
\begin{array}{r}
\min _{x, y \in N(z) \backslash\{p, q\}} \max \{l(x z)+l(z p)-l(x p), l(y z)+l(z p)-l(y p), \\
l(x z)+l(z q)-l(x q), l(y z)+l(z q)-l(y q)\} .
\end{array}
$$

Theorem 3 (Strong Close Point Elimination Theorem). Let $p q$, pr and $r x$ be three edges of a TSP instance $(V, E)$. Let $z \in V \backslash\{p, q, r, x\}$. If

$$
l(x q)+l(r z)+l(z p)-m_{p r}(z)<l(p q)+l(r x),
$$

then the edges $p q$, pr and $r x$ are 3-incompatible.

Proof. Assume that an optimum tour $T$ contains the edges $p q, p r$ and $r x$. We show that there exists a 3-opt move yielding a tour shorter than $T$. Delete the edges $p q$ and $r x$ and insert the edges $q x, p z$ and $r z$. Note that this edge set is eulerian but not a TSP tour as vertex $z$ has degree four. But as $l(x q)+l(r z)+$ $l(z p)-m_{p r}(z)<l(p q)+l(r x)$ a short cut is possible that yields a tour shorter than $T$. This contradicts the optimality of the tour $T$. 


\section{$5 \quad$ Certifying potential points}

Starting with a complete graph, it can be very inefficient, to exclude edges using the criteria discussed so far. We will show how to adopt the Main Edge Elimination Theorem such that most edges can be excluded efficiently.

The aim of this section is to show that one can prove in constant time that a point $r$ is potential with respect to an edge $p q$. To be able to do so, we will assume in the following that the distance function $l$ is the EUC_2D function. Similar results hold for any other distance function that is close to the Euclidean distance. By $|p q|$ we denote the Euclidean distance of $p$ and $q$. Note that

$$
l(p q)-\frac{1}{2} \leq|p q| \leq l(p q)+\frac{1}{2} .
$$

We now want to find a covering for a point $r$ and an edge $p q$. We will therefore take a closer look at the set $R:=\{x \in V \mid r x \in E \wedge p q \sim r x\}$ which was already defined in Section 3. Observe that in a metric space the compatibility of the edges $p q$ and $r s$ implies that the edges $p q$ and $r t$ are also compatible for all $t \in r s$. Since we use EUC_2D lengths, this only holds after adding some constants. For each point $r$ choose $\delta_{r}$ s.t. no vertex apart from $r$ lies in the interior of the circle around $r$ with radius $\delta_{r}$. One can for example use

$$
\delta_{r}:=\frac{1}{2}+\max \left\{d \in \mathbb{Z}_{+} \mid \forall s \in V \backslash\{r\} l(r s)>d\right\} .
$$

For an edge $p q$ and a point $r \in V \backslash\{p, q\}$ define the two lengths

$$
l_{p}:=\delta_{r}+l(p q)-l(q r)-1 \quad \text { and } \quad l_{q}:=\delta_{r}+l(p q)-l(p r)-1 .
$$

For each point $s \in V \backslash\{r\}$ define $s_{r} \in r s$ such that $\left|r s_{r}\right|=\delta_{r}$.

Lemma 2. Let $(V, E)$ be a TSP instance, $p q \in E, r \in V \backslash\{p, q\}$ and $s \in V \backslash\{r\}$. If $\left|p s_{r}\right|<l_{p}$ and $\left|q s_{r}\right|<l_{q}$ then the edges $p q$ and rs are incompatible.

Proof. We show that both 2-opt moves involving the edges $p q$ and $r s$ have shorter length. Using (9), (11), and the triangle inequality we get:

$$
\begin{aligned}
l(p s)+l(q r) & \leq\left|p s_{r}\right|+\left|s s_{r}\right|+\frac{1}{2}+l(q r)<l_{p}+\left|s s_{r}\right|+\frac{1}{2}+l(q r) \\
& =\delta_{r}+l(p q)-l(q r)-\frac{1}{2}+\left|s s_{r}\right|+l(q r) \leq l(p q)+l(r s)
\end{aligned}
$$

$l(q s)+l(p r)<l(p q)+l(r s)$ is proven analogously. Hence $p q$ and $r s$ are incompatible.

Figure 2 illustrates Lemma 2. It shows two cones $R_{p}$ and $R_{q}$ for which $R \subset$ $R_{p} \cup R_{q}$. The cones are defined as $R_{p}:=\left\{t|| q t_{r} \mid \geq l_{q}\right\}$ and $R_{q}:=\left\{t|| p t_{r} \mid \geq l_{p}\right\}$. We do not need that the cones $R_{p}$ and $R_{q}$ are disjoint. However we need that they actually exist, i.e. the circles around $p$ and $q$ have to intersect the circle around $r$. 


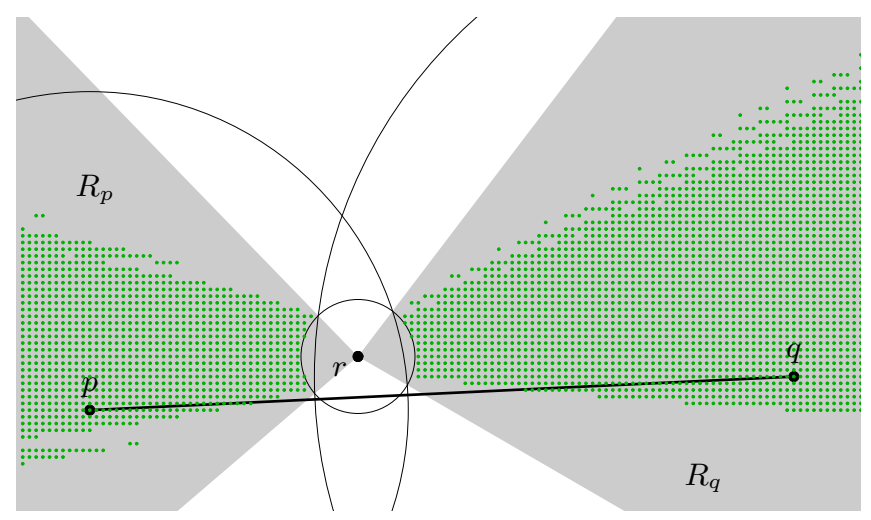

Fig. 2. The small dots indicate all possible endpoints for the edge $r s$ such that $r s \sim p q$. By Lemma 2 all these vertices must be contained in the gray cones $R_{p}$ and $R_{q}$.

Lemma 3. Let $(V, E)$ be a TSP instance, $p q \in E$ and $r \in V \backslash\{p, q\}$. If

$$
l_{p}+l_{q} \geq l(p q)-\frac{1}{2}
$$

then the circle with center $r$ and radius $\delta_{r}$ intersects both circles with centers $p$ and $q$ and radii $l_{p}$ respectively $l_{q}$.

Proof. It suffices to show $|i r|-\delta_{r} \leq l_{i} \leq|i r|+\delta_{r}$ for $i \in\{p, q\}$.

$$
\begin{aligned}
|p r|-\delta_{r} & \leq l(p r)+\frac{1}{2}-\delta_{r} \stackrel{110}{=} l(p q)-\frac{1}{2}-l_{q} \stackrel{\sqrt[12]{\leq}}{\leq} l_{p} \\
& \stackrel{111}{\leq}\left(|p r|+|q r|+\frac{1}{2}\right)-\left(|q r|-\frac{1}{2}\right)+\delta_{r}-1=|p r|+\delta_{r}
\end{aligned}
$$

Analogously for $i=q$.

A naive way to prove that $R_{p}$ and $R_{q}$ certify the potentiality of $r$ is to look at all possible 3 -opt moves.

Lemma 4. Let $(V, E)$ be a TSP instance and $p q \in E$. For $r \in V \backslash\{p, q\}$ let $R \subset R_{p} \cup R_{q}$ with $R=\{x \in V \mid r x \in E \wedge p q \sim r x\}$. If for $i \in\{p, q\}$ :

$$
l(p q)+l(r x)+l(r y)>l(p r)+l(r q)+l(x y) \quad \text { for all } x, y \in R_{i},
$$

then $R_{p}$ and $R_{q}$ certify the potentiality of $r$.

Proof. Assume that $p q$ is contained in an optimum tour $T$ and $r x, r y \in T$ with $x, y \in R_{i}$ for $i \in\{p, q\}$. Then replacing the edges $p q, r x$ and $r y$ by the edges $p r, r q$ and $x y$ is a valid 3-opt move. By inequality (13) this 3-opt move yields a shorter tour, contradicting the optimality of $T$. 
Lemma 4 yields a method to check in $O\left(n^{2}\right)$ time that a vertex $r$ is potential. We now want to show that the potentiality of a vertex $r$ can be certified in constant time. We assume that the edge $p q$ is part of an optimum tour $T$. Furthermore we consider the covering $R_{p}$ and $R_{q}$ as described above. The angles of the cones $R_{p}$ and $R_{q}$ are denoted by $\alpha_{p}$ respectively $\alpha_{q}$. The certification follows from a simple argument.

Lemma 5. Assume that $p q$ is contained in an optimum TSP tour T, $r \in V \backslash$ $\{p, q\}$ and the angle $\gamma$ between the two edges incident with $r$ in $T$ satisfies

$$
\gamma>\max \left\{\alpha_{p}, \alpha_{q}\right\}
$$

Then the neighbors of $r$ in $T$ cannot both lie in $R_{p}$ respectively $R_{q}$.

Proof. W.l.o.g. assume both neighbors of $r$ in $T$ lie in $R_{p}$. This immediatly implies $\gamma \leq \alpha_{p}$, contradicting (14).

It now suffices to show that inequality (14) holds for every optimum tour containing $p q$. This can be checked using the following statement.

Lemma 6. Let $(V, E)$ be a TSP instance and $T$ an optimum tour. Let $p q \in T$ and $r \in V \backslash\{p, q\}$. Assume that inequality (12) holds. Define the angle $\gamma_{r}$ as

$$
\gamma_{r}:=\arccos \left(1-\frac{\left(l_{p}+l_{q}-l(p q)+\frac{1}{2}\right)^{2}}{2 \delta_{r}^{2}}\right) .
$$

Then the angle $\gamma$ between the two edges of $T$ incident with vertex $r$ satisfies

$$
\gamma \geq \gamma_{r}
$$

Proof. Let $r x, r y \in T$ be the two incident edges of $r$. Let $\mu:=\left|x_{r} y_{r}\right|$. The cosine formula yields the following equation:

$$
\mu^{2}=2 \delta_{r}^{2}-2 \delta_{r}^{2} \cos \gamma
$$

As $T$ is an optimum tour, there is no valid 3-opt move which yields a shorter tour. Hence we get:

$$
\begin{aligned}
& l(p q)+l(r x)+l(r y) \leq l(p r)+l(q r)+l(x y) \\
& \Rightarrow \quad l_{p}+l_{q}+\left|x_{r} x\right|+\left|y_{r} y\right|-l(p q)+1 \leq l(x y) \\
& \Rightarrow \quad l_{p}+l_{q}-l(p q)+\frac{1}{2} \leq \mu \\
& \stackrel{12}{\Rightarrow} \quad\left(l_{p}+l_{q}-l(p q)+\frac{1}{2}\right)^{2} \leq \mu^{2}=2 \delta_{r}^{2}-2 \delta_{r}^{2} \cos \gamma \\
& \Rightarrow \quad \cos \gamma \leq 1-\frac{\left(l_{p}+l_{q}-l(p q)+\frac{1}{2}\right)^{2}}{2 \delta_{r}^{2}} \\
& \Rightarrow \quad \gamma \geq \gamma_{r} .
\end{aligned}
$$


From Lemma 5] and Lemma [6] we immediately get the following result.

Lemma 7. Let $p q$ be an edge contained in some optimum TSP tour $T$ and $r \in V \backslash\{p, q\}$. Assume that inequality (12) holds. If

$$
\gamma_{r}>\max \left\{\alpha_{p}, \alpha_{q}\right\}
$$

then the sets $R_{p}$ and $R_{q}$ certify the potentiality of $r$.

It is easy to see that the angles $\alpha_{p}$ and $\alpha_{q}$ can be computed in constant time. Details are given in the appendix.

The results so far provide a way to prove in constant time that a given vertex is potential. Notice that not all potential points can be detected using this approach. However, as we will see in Section [7 sufficiently many potential points can be detected using this method. For simplifying notation we introduce the following concept: Let $p q$ be an edge and $r \in V \backslash\{p, q\}$. The vertex $r$ is called strongly potential (with respect to $p q$ ), if the conditions (12) and (18) hold.

Thus, checking whether a point $r$ is strongly potential can be done in constant time (assuming that the value $\delta_{r}$ is known, which can be computed in a preprocessing step for all vertices). Verifying the inequalities in the Main Edge Elimination Theorem still needs $O(n)$ time. The aim now is to show that this can be done in constant time by computing appropriate lower bounds for (3) and (4).

Lemma 8. Let $(V, E)$ be a TSP instance and $r$ strongly potential with respect to $p q$. Let $R_{p}$ and $R_{q}$ be the covering certifying $r$. Then

$$
\begin{aligned}
& \min _{x \in R_{p}}\{l(r x)-l(p x)\} \geq \delta_{r}-1-\max \left\{\left|p x_{r}\right|: x \in R_{p}\right\} \text { and } \\
& \min _{y \in R_{q}}\{l(r y)-l(q y)\} \geq \delta_{r}-1-\max \left\{\left|q y_{r}\right|: y \in R_{q}\right\} .
\end{aligned}
$$

Proof. Let $x \in R_{p}$. Then

$$
\begin{aligned}
l(r x)-l(p x) & \geq|r x|-|p x|-1 \geq \delta_{r}+\left|x_{r} x\right|-\left(\left|p x_{r}\right|+\left|x_{r} x\right|\right)-1 \\
& \geq \delta_{r}-1-\max \left\{\left|p x_{r}\right|: x \in R_{p}\right\}
\end{aligned}
$$

Similarly one can prove this for the set $R_{q}$.

Let $C_{r}$ be the circle around $r$ with radius $\delta_{r}$. Define the two arcs

$$
B_{p}:=\left\{x \in C_{r}|| q x \mid \geq l_{q}\right\} \quad \text { and } \quad B_{q}:=\left\{y \in C_{r}|| p y \mid \geq l_{p}\right\} .
$$

Further let $\tilde{p}$ and $\tilde{q}$ be the points on $C_{r}$ with greatest distance to $p$ respectively $q$. Since $B_{p}$ and $B_{q}$ are connected, the maxima in the inequalities (19) and (20) can only be attained at $\tilde{p}$ respectively $\tilde{q}$, or at the endpoints of $B_{p}$ respectively $B_{q}$. We consider the case that

$$
|p \tilde{q}| \leq l_{p} \quad \text { and } \quad|q \tilde{p}| \leq l_{q} .
$$


This gives that

$$
\begin{aligned}
\max \left\{\left|p x_{r}\right|: x \in R_{p}\right\} & \leq \max \left\{t \mid t \in B_{p}\right\} \quad \text { and } \\
\max \left\{\left|q y_{r}\right|: y \in R_{q}\right\} & \leq \max \left\{t \mid t \in B_{q}\right\},
\end{aligned}
$$

where the right hand sides can easily be calculated in constant time. Details are given in the appendix.

\section{The Algorithm}

In this section we describe our algorithm that eliminates useless edges. It consists of three independent steps. Step 1 uses the results of Section 3 and Section 5 and eliminates the majority of all edges. Step 2 applies the Main Edge Elimination in combination with the Close Point Elimination to eliminate additional edges. Finally in Step 3 we use a backtrack search of bounded depth to eliminate even more edges.

Step 1: Fast Elimination To prove that an edge $p q$ is useless we need to find two potential points $r$ and $s$ satisfying the conditions of the Main Edge Elimination Theorem. For a point $r$ we use the method described in Section 5 , to prove that it is potential. In fact we will only use $r$ if it is strongly potential. This can be checked in constant time. The next step is to calculate the minima appearing in the Main Edge Elimination Theorem using Lemma 8, This can be done separately for each potential point in constant time.

Once two potential points $r$ and $s$ with their corresponding minima are calculated, one can check in constant time whether the inequalities of the Main Edge Elimination Theorem are satisfied. Since only two potential points which satisfy the conditions of the theorem are needed, a smart ordering and stopping criteron for checking the potentiality of points can speed up the algorithm drastically. We select the points ordered by their distance from the midpoint of the edge $p q$ and stop after at most 10 points that have been considered.

Using a 2-d tree 2] we compute in a preprocessing step the values $\delta_{r}$ for all vertices of the instance. In most cases it turns out that at most three strongly potential points have to be considered to prove that an edge is useless.

Step 2: Direct Elimination For an edge $p q$ we consider two vertices $r$ and $s$ with all their incident edge pairs. The Close Point Elimination is used to reduce the number of possible edge pairs. The edge $p q$ can be eliminated either if no edge pair can be found for $r$ or $s$, or if all combinations of edge pairs satisfy the condition of the Main Edge Elimination Theorem.

Step 3: Backtrack Search In this step starting with an edge $p q$ we extend a set of disjoint paths recursively. We allow two operations for the extension. Either we select one of the existing paths and add an edge incident to one of 
its endpoints. Or we add a vertex not yet contained in any of the disjoint paths and two edges incident with this vertex. After each extension we check whether the Main Edge Elimination Theorem or the Close Point Elimination allows to eliminate one of the path edges. In this case we backtrack. Moreover we check that the collection of paths is minimal in the sense that no collection of paths exists that has shorter length and that connects the same pairs of endpoints and uses the same set of interior points. We use an extension of the Held-Karp algorithm [6] for this. We always select the extension of the set of paths that has the smallest number of possibilities. If all extensions have been examined without reaching a predefined extension depth, then we have proven that edge $p q$ is useless.

\section{$7 \quad$ Results}

We applied our algorithm to all TSPLIB 9] instances which use the EUC_2D metric as well as to some larger EUC_2D instances from [3]. Table 1] contains the results on some of these instances.

Table 1. Results for some TSPLIB instances with at least 1,000 vertices as well as a 100,000 vertex instance from [3. The first three columns contain the instance name, the number of vertices and the number of edges. Then for each of the three steps as described in Section [6 we list the number of edges that remain after this step as well as the runtime. For Step 3 we used an extension depth of 10 which gave a reasonable trade off between the runtime and the number of eliminated edges. The last two columns contain the total runtime of our algorithm and the ratio of the number of edges remaining after Step 3 divided by the number of vertices. All runtimes are given in the format $\mathrm{hh}: \mathrm{mm}: \mathrm{ss}$ and are measured on a single core of a $2.9 \mathrm{GHz}$ Intel Xeon.

\begin{tabular}{|c|c|c|c|c|c|c|c|c|c|c|}
\hline \multirow{2}{*}{ instance } & \multirow{2}{*}{$n$} & \multirow[b]{2}{*}{$m$} & \multicolumn{2}{|c|}{ Step 1} & \multicolumn{2}{|c|}{ Step 2} & \multicolumn{2}{|c|}{ Step 3} & \multirow{2}{*}{$\begin{array}{r}\text { total } \\
\text { runtime }\end{array}$} & \multirow{2}{*}{ ratio } \\
\hline & & & edges & time & edges & time & edges & time & & \\
\hline$\overline{\mathrm{r} 1002}$ & 1002 & 501501 & 42636 & 1 & 5810 & $2: 13$ & 4521 & $2: 28: 07$ & $2: 30: 21$ & 4.2 \\
\hline u1060 & 1060 & 561270 & 43887 & 1 & \begin{tabular}{|c|}
6063 \\
\end{tabular} & $2: 24$ & 4619 & $3: 30: 48$ & $3: 33: 13$ & $\overline{4.4}$ \\
\hline vm1084 & 1084 & 586986 & 40958 & 1 & 6035 & $3: 28$ & 4610 & $1: 17: 35$ & $1: 21: 05$ & $\overline{4.3}$ \\
\hline pcb1173 & 1173 & 687378 & 32533 & 1 & 7662 & 33 & 6084 & $3: 10: 17$ & $3: 10: 51$ & $\overline{5.2}$ \\
\hline d1291 & 1291 & 832695 & 122897 & 4 & \begin{tabular}{l|l|}
12552 \\
\end{tabular} & $52: 21$ & 11317 & $13: 33: 14$ & $14: 25: 40$ & 8.8 \\
\hline rl1304 & 1304 & 849556 & 124561 & 3 & 21689 & $11: 54$ & 14527 & $12: 53: 14$ & $13: 05: 11$ & 11.1 \\
\hline rl1323 & 1323 & 874503 & 106860 & 2 & 16743 & $5: 33$ & 12691 & $9: 41: 18$ & $9: 46: 53$ & 9.6 \\
\hline nrw1379 & 1379 & 950131 & 28468 & 1 & \begin{tabular}{|l|}
7199 \\
\end{tabular} & $1: 58$ & 5752 & $2: 12: 44$ & $2: 14: 44$ & $\overline{4.2}$ \\
\hline u1432 & 1432 & 1024596 & 21970 & 1 & 7817 & $2: 51$ & 6495 & $2: 13: 02$ & $2: 15: 55$ & 4.5 \\
\hline d1655 & 1655 & 1368685 & 230855 & 9 & 14345 & $37: 30$ & 12103 & 10:05:46 & $10: 43: 26$ & 7.3 \\
\hline $\mathrm{vm} 1748$ & 1748 & 1526878 & 144681 & 6 & 12303 & $23: 10$ & 7691 & $2: 48: 14$ & $3: 11: 30$ & $\overline{4.4}$ \\
\hline u1817 & 1817 & 1649836 & 109056 & 5 & 13201 & $10: 58$ & 11736 & $6: 19: 22$ & $6: 30: 25$ & 6.5 \\
\hline rl1889 & 1889 & 1783216 & 206768 & 9 & 23410 & $3: 15: 40$ & 18673 & $25: 18: 52$ & $28: 34: 41$ & 9.9 \\
\hline $\mathrm{d} 2103$ & 2103 & 2210253 & 166866 & 8 & 19631 & $55: 01$ & 18105 & $18: 19: 34$ & $19: 14: 44$ & 8.6 \\
\hline u2152 & 2152 & 2314476 & 117030 & 5 & 15101 & $11: 17$ & 13170 & $7: 07: 45$ & $7: 19: 08$ & 6.1 \\
\hline $\mathrm{u} 2319$ & 2319 & 2687721 & 21698 & 3 & 9919 & 44 & 9473 & $1: 41: 41$ & $1: 42: 22$ & 4.1 \\
\hline pr2392 & 2392 & 2859636 & 121514 & 7 & $\begin{array}{ll}15598 \\
\end{array}$ & 13:03 & 12088 & $7: 41: 45$ & $7: 44: 55$ & 5.1 \\
\hline pcb3038 & 3038 & 4613203 & 95576 & 8 & \begin{tabular}{l|l}
17940 \\
\end{tabular} & $11: 05$ & 14869 & $5: 44: 08$ & $5: 55: 22$ & 4.9 \\
\hline fnl4461 & 4461 & 9948030 & 128527 & 15 & 23963 & $9: 30$ & 19082 & $7: 14: 21$ & $7: 24: 07$ & $\overline{4.3}$ \\
\hline brd14051 & 14051 & 98708275 & 2661869 & $4: 39$ & 93497 & $18: 50: 15$ & 64486 & $28: 39: 22$ & $47: 34: 16$ & $\overline{4.6}$ \\
\hline $\mathrm{d} 15112$ & 15112 & 114178716 & 1703765 & $5: 51$ & \begin{tabular}{|l|}
130110 \\
\end{tabular} & $10: 25: 44$ & 66010 & $38: 37: 42$ & 49:09:17 & 4.4 \\
\hline $\mathrm{d} 18512$ & 18512 & 171337816 & 1449877 & 5:30| & 112681 & $1: 49: 35$ & 84203 & $32: 38: 07$ & $34: 33: 13$ & 4.5 \\
\hline mona-lisa100k & 100000 & 4999950000 & 2071297 & $3: 45: 21$ & 476001 & $22: 51$ & 322716 & $55: 42: 51$ & $59: 51: 04$ & 3.2 \\
\hline
\end{tabular}


The TSP solver Concorde [1] is the fastest available algorithm to solve large TSP instances optimally. It can be downloaded at $[3$. We applied Concorde to the TSPLIB instance d2103. The total runtime needed by Concorde was $17,219,190$ second\$1 This agrees with the runtime reported for this instance on page 503 of [1]. Then we ran the three steps of our algorithm as described in Section 6, For Step 3 we used an extension depth of 12 . After 168, 153 seconds all but 16,566 edges were eliminated. We changed the length of all eliminated edges to some large value and gave this new instance again to Concorde. On this instance Concorde needed 1,392,582 seconds. Thus the total runtime was improved by our edge elimination algorithm by more than a factor of 11 .

Two other successful applications of our edge elimination approach were reported to us by Cook [4. First, the edge elimination approach in combination with the LKH algorithm [7 improved the so far best known TSP tour for the DIMACS instance E100k.0 [8]. The shortest tour known so far had length $225,786,982$. It was found using the LKH algorithm. Cook's implementation of an edge elimination approach eliminated all but 274,741 edges in the TSP instance E100k.0. By applying the LKH algorithm to this edge set a tour of length 225,784,127 was found [8. Secondly, Cook applied the edge elimination approach to a truly Euclidean instance (i.e., a Euclidean instance where the point distances are not rounded). Finding optimum TSP tours in such instances is much harder than in instances with rounded Euclidean norm. The largest truly Euclidean instance that Cook was able to solve so far had 500 points. With the help of the edge elimination approach he solved an instance with 1000 points.

\section{References}

1. D.L.Applegate, R.E.Bixby, V.Chvátal, W.J.Cook. The Traveling Salesman Problem. A Computational Study. Princeton University Press, 2006

2. J.L.Bently. Multidimensional binary search trees used for associative searching. Communications of the ACM, Volume 18, Number 9 (September 1975), pp. 509-517

3. W.J.Cook's TSP website at http://www.math.uwaterloo.ca/tsp/

4. W.J.Cook, personal communication, December 2013.

5. M.R.Garey, D.S.Johnson. Computers and Intractability. Freeman, New York 1979

6. M.Held, R.M.Karp A Dynamic Programming Approach to Sequencing Problems. Journal of the Society for Industrial and Applied Mathematics, Vol. 10, No. 1 (Mar.,1962), pp. 196-210

7. K.Helsgaun. General $k$-opt submoves for the Lin-Kernighan TSP heuristic. Mathematical Programming Computation, Volume 1, Issue 2-3 (2009), pp. 119-163

8. K.Helsgaun, http://www.akira.ruc.dk/ ${ }^{\circ}$ keld/research/LKH/DIMACS_results.html

9. G.Reinelt. TSPLIB 95. Interdisziplinäres Zentrum für Wissenschaftliches Rechnen (IWR), Heidelberg, 1995

\footnotetext{
${ }^{1}$ Runtime on a $2.9 \mathrm{GHz}$ Intel Xeon using Concorde 3 . We observed that the runtime of Concorde can vary by more than $40 \%$ on the same instance. Therefore, we took the average runtime of two independent runs. The Concorde log-files for all runs can be found at http://www.or.uni-bonn.de/ ^hougardy/EdgeElimination.
} 


\section{Appendix}

\subsection{The angles $\alpha_{p}$ and $\alpha_{q}$}

The following lemma shows how to compute the angles $\alpha_{p}$ and $\alpha_{q}$, discussed in Section 5 .

Lemma 9. Let $(V, E)$ be TSP instance, $p q \in E$ and $r \in V \backslash\{p, q\}$. Then the angles $\alpha_{p}$ and $\alpha_{q}$ of the cones $R_{p}$ respectively $R_{q}$ satisfy

$$
\begin{aligned}
& \alpha_{p}=2 \cdot \arccos \left(\frac{l_{q}^{2}-\delta_{r}^{2}-|r q|^{2}}{2 \delta_{r}|r q|}\right) \quad \text { respectively } \\
& \alpha_{q}=2 \cdot \arccos \left(\frac{l_{p}^{2}-\delta_{r}^{2}-|r p|^{2}}{2 \delta_{r}|r p|}\right) .
\end{aligned}
$$

Proof. Let $C_{r}$ be the circle around $r$ with radius $\delta_{r}$ and $C_{q}$ the circle around $q$ with radius $l_{q}$. Let $t$ be one intersection point of these two circles. Consider the triangle with the points $q, r$ and $t$. Let $\omega_{q}$ be the angle of the triangle at the point $r$. The cosine theorem then yields

$$
\delta_{r}^{2}+|r q|^{2}-2 \delta_{r}|r q| \cos \left(\omega_{q}\right)=l_{q}^{2} .
$$

Hence we can conclude

$$
\begin{aligned}
\cos \left(\frac{\alpha_{p}}{2}\right) & =\cos \left(180^{\circ}-\omega_{q}\right) \\
& =-\cos \left(\omega_{q}\right) \\
& \stackrel{26}{=} \frac{l_{q}^{2}-\delta_{r}^{2}-|r q|^{2}}{2 \delta_{r}|r q|} .
\end{aligned}
$$

This equation implies (24). Equation (25) can be shown equivalently.

\subsection{Validating}

In order to calculate the maxima of (19) and (20), we need to define some angles. For an edge $p q \in E$ and a point $r \in V \backslash\{p, q\}$ we define $\varepsilon_{p}$ and $\varepsilon_{q}$ as the angles in the triangle $p q r$ at the points $p$ respectively $q$. Further let $t$ be such that $|r t|=\delta_{r}$ and $|p t|=l_{p}$. Let $\theta_{p}$ be the angle in the triangle $p r t$ at the point $p$. The angle $\theta_{q}$ is defined equivalently. By the cosine theorem we obtain the following equations:

$$
\begin{aligned}
\cos \varepsilon_{p} & =\frac{|p q|^{2}+|p r|^{2}-|q r|^{2}}{2|p q||p r|} \\
\cos \varepsilon_{q} & =\frac{|p q|^{2}+|q r|^{2}-|p r|^{2}}{2|p q||q r|} \\
\cos \theta_{p} & =\frac{l_{p}^{2}+|p r|^{2}-\delta_{r}^{2}}{2 l_{p}|p r|} \\
\cos \theta_{q} & =\frac{l_{q}^{2}+|q r|^{2}-\delta_{r}^{2}}{2 l_{q}|q r|} .
\end{aligned}
$$




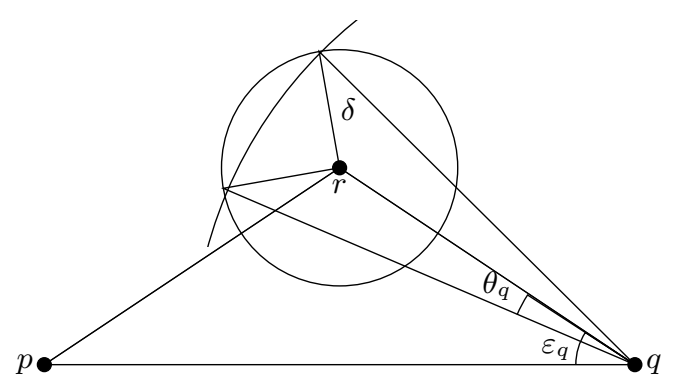

Fig. 3. The angles $\varepsilon$ and $\theta$

By considering the cosine theorem in the triangles $p q \tilde{p}$ respectively $p q \tilde{q}$, the inequalities (21) are equivalent to

$$
\begin{aligned}
& \frac{\left(|q r|+\delta_{r}\right)^{2}+|p q|^{2}-l_{p}^{2}}{2\left(|q r|+\delta_{r}\right)|p q|} \leq \cos \varepsilon_{q} \\
& \frac{\left(|p r|+\delta_{r}\right)^{2}+|p q|^{2}-l_{q}^{2}}{2\left(|p r|+\delta_{r}\right)|p q|} \leq \cos \varepsilon_{p} .
\end{aligned}
$$

We want to calculate the maxima of Lemma 8 . They correspond to

$$
\begin{aligned}
& \max \left\{\left|p s_{r}\right|: s \in R_{p}\right\}=\max \left\{b_{p}, b_{p}^{\prime}\right\} \\
& \max \left\{\left|q s_{r}\right|: s \in R_{q}\right\}=\max \left\{b_{q}, b_{p}^{\prime}\right\},
\end{aligned}
$$

whereas the endpoints of the archs $B_{p}$ and $B_{q}$ are defined as $b_{p}$ and $b_{p}^{\prime}$ respectively $b_{q}$ and $b_{q}^{\prime}$.

Lemma 10. Assuming $\varepsilon \leq 180^{\circ}$ and $\theta \leq 180^{\circ}$ then we have the following equivalence:

$$
\varepsilon+\theta \leq 180^{\circ} \Leftrightarrow \cos \varepsilon+\cos \theta \geq 0
$$

Using simple geometric calculations, we can deduce the following lemma:

Lemma 11. Let $r$ be strongly potential with respect to $p q$, and let the inequalities (21) hold. Let $\varepsilon_{p}+\theta_{p} \leq 180^{\circ}$ and $\varepsilon_{q}+\theta_{q} \leq 180^{\circ}$. Then

$$
\begin{aligned}
& \left(\max \left\{\left|p s_{r}\right|: s \in R_{p}\right\}\right)^{2}=|p q|^{2}+l_{q}^{2}-2|p q| l_{q} \cos \left(\varepsilon_{q}+\theta_{q}\right) \\
& \left(\max \left\{\left|q s_{r}\right|: s \in R_{q}\right\}\right)^{2}=|p q|^{2}+l_{p}^{2}-2|p q| l_{p} \cos \left(\varepsilon_{p}+\theta_{p}\right)
\end{aligned}
$$

Proof. From the assumption we can follow $\cos \left(\varepsilon_{q}-\theta_{q}\right) \geq \cos \left(\varepsilon_{q}+\theta_{q}\right)$. The cosine theorem then implies

$$
\left(\max \left\{\left|p s_{r}\right|: s \in R_{p}\right\}\right)^{2}=|p q|^{2}+l_{q}^{2}-2|p q| l_{q} \cos \left(\varepsilon_{q}+\theta_{q}\right) .
$$

